\title{
Harnack Inequality and No-Arbitrage Analysis
}

\author{
Wanxiao Tang ${ }^{\dagger}$, Fanchao Zhou ${ }^{\dagger}$ and Peibiao Zhao *i] \\ Department of Mathematics, Nanjing University of Science and Technology, Nanjing 210094, China; \\ wanxiaotang92@163.com (W.T.); fczhou01forp@163.com (F.Z.) \\ * Correspondence: pbzhao@njust.edu.cn \\ + These authors contributed equally to this work.
}

Received: 13 August 2018; Accepted: 15 October 2018; Published: 16 October 2018

Abstract: The present paper attains a Harnack inequality for the option pricing (or Kolmogorov) equation with gradient estimate arguments. We then perform a no-arbitrage analysis of a financial market.

Keywords: Harnack inequality; fundamental solution; no arbitrage

\section{Introduction}

The study of Harnack inequality has been an active field in the past three decades. Harnack inequality states that the values of the nonnegative solution to a harmonic function are comparable. There are many articles presenting the Harnack inequality through various equations in European space or Riemannian manifold [1,2]. Additionally, some scholars study its applications [3-5].

In finance, the no-arbitrage hypothesis is an important theoretical basis in studying a series of financial problems (e.g., the investment portfolio and the option pricing theory). Many articles have studied the no-arbitrage condition mainly by functional analysis, the Martingale approach, and convex optimization. These articles yield plentiful rich results [6-8].

In recent years, new perspectives have appeared in researching current problems in the financial market. Romeil Sandhu [9] characterized the market fragility by Ricci curvature. Brody [10] applied information geometry to the theory of interest rates to analyze the relationship of two different yield curves. Young [11], Kirill Ilinski [12], and Simone Farinelli [13] characterized the geometric no-arbitrage condition by the curvature. Yang Ho Choi [14] derived a general asymptotic solution of the multidimensional Black-Scholes formula without the constant volatility assumption by the heat kernel expansion. Carciola et al. [3] proved the Harnack inequality of option pricing (or Kolmogorov operator) equations based on the fundamental solution of the Kolmogorov operator equations and studied the no-arbitrage bounds for a financial market by using this Harnack inequality.

Motivated by the work of Carciola et al. [3], a natural question is that, if the fundamental solution is not available, can one consider and study similar problems? From this consideration, we consider a similar no-arbitrage problem to some Kolmogorov-like operator equations based on a Harnack inequality with gradient estimate arguments instead of the argument of a fundamental solution for a financial market.

The organization of this paper is as follows. We state some necessary terminologies and notations in Section 2. Section 3 is devoted to perform the Harnack inequality and no-arbitrage analysis. Conclusions are provided in Section 4. 


\section{Preliminaries}

For convenience, we first state some necessary terminologies and notations as follows.

Consider a market with $n$ stocks $S_{t}^{i}(i=1,2, \cdots, n)$ and a risk-free asset $B_{t}$, such as a bank account, at the time $t$. Suppose the prices of the stocks and the bank account are described by

$$
d S_{t}^{i}=r S_{t}^{i} d t+S_{t}^{i} \sum_{j=1}^{n} \sigma_{i j} d W_{t}^{j}=r S_{t}^{i} d t+\sigma^{i} S_{t}^{i} d W_{t}
$$

and

$$
d B_{t}=r B_{t} d t
$$

where $W=\left(W^{1}, \cdots, W^{n}\right)$ is a standard $n$-dimensional Brownian motion, $\sigma$ is a non-singular $n \times n$ matrix and the risk-free rate $r$ is a constant.

Consider a portfolio described by a process $\theta=\left(\alpha_{t}, \beta_{t}\right)=\left(\alpha^{1}, \ldots, \alpha^{n}, \beta\right)$, where $\alpha_{t}=\alpha\left(t, S_{t}\right)$ and $\beta_{t}=\beta\left(t, S_{t}\right)$ are smooth functions. The portfolio value can be expressed as

$$
V_{t}=\sum_{i=1}^{n} \alpha_{t}^{i} S_{t}^{i}+\beta_{t} B_{t}=\alpha_{t} S_{t}+\beta_{t} B_{t}
$$

A strategy is called self-financing if

$$
d V_{t}=\alpha_{t} d S_{t}+\beta_{t} d B_{t}
$$

Construct a portfolio $\Pi_{t}$ : buy one option $C\left(S^{1}, \ldots, S^{n}, t\right)$, which is a function of $S^{i}$ and $t$, and sell $\triangle_{i}$ underlying assets $S^{i}$. The value of the portfolio is then $\Pi_{t}=C\left(S^{1}, \cdots, S^{n}, t\right)-\sum_{i=1}^{n} \triangle_{i} S_{t}^{i}$, where $\triangle_{i}$ can be chosen suitably so that $\Pi_{t}$ is risk-free. Thus, the portfolio $\Pi_{t}$ can be described by

$$
d \Pi_{t}=r \Pi_{t} d t
$$

where $r$ is a risk-free rate, that is,

$$
d \Pi_{t}=d C-\sum_{i=1}^{n} \triangle_{i} d S^{i}=r \Pi_{t} d t=r\left(C-\sum_{i=1}^{n} \triangle_{i} S^{i}\right) d t .
$$

Next we apply the Itô formula to $C$, so that we have

$$
d C=\frac{\partial C}{\partial t} d t+\sum_{i=1}^{n} \frac{\partial C}{\partial S^{i}} d S^{i}+\frac{1}{2} \sum_{i, j=1}^{n} \frac{\partial^{2} C}{\partial S^{i} \partial S^{j}} d S^{i} d S^{j}
$$

Thus,

$$
\begin{aligned}
& \left(\frac{\partial C}{\partial t}+\frac{1}{2} \sum_{i, j=1}^{n}\left(\sigma \sigma^{*}\right)_{i j} S^{i} S^{j} \frac{\partial^{2} C}{\partial S^{i} \partial S^{j}}\right) d t+\sum_{i=1}^{n}\left(\frac{\partial C}{\partial S^{i}}-\triangle_{i}\right) d S^{i} \\
& =r\left(C-\sum_{i=1}^{n} \triangle_{i} S^{i}\right) d t
\end{aligned}
$$

where $\sigma^{*}$ is the transpose of $\sigma$. The right-hand side of Equation (2) is risk-free, so the coefficient of the stochastic term on the left-hand side should be zero. We can take $\triangle_{i}=\frac{\partial C}{\partial S^{i}}$ and remove $d t$. Thus, we arrive at

$$
\frac{\partial C}{\partial t}+\frac{1}{2} \sum_{i, j=1}^{n}\left(\sigma \sigma^{*}\right)_{i j} S^{i} S^{j} \frac{\partial^{2} C}{\partial S^{i} \partial S^{j}}+\sum_{i=1}^{n} r S^{i} \frac{\partial C}{\partial S^{i}}-r C=0
$$


It is well-known that Equation (1) is equivalent to the fact that $V_{t}=V\left(t, S_{t}\right), \alpha_{t}=\nabla_{S} V\left(t, S_{t}\right)$ and $\beta_{t}=e^{-r t}\left(V\left(t, S_{t}\right)-S_{t} \nabla_{S} V\left(t, S_{t}\right)\right)$, where $V=V\left(t, S_{t}\right)$ is a solution to the $B-S$ equation:

$$
\frac{1}{2} \sum_{i, j=1}^{n}\left(\sigma \sigma^{*}\right)_{i j} S^{i} S^{j} \partial_{S^{i} S^{j}} V+r S \nabla_{S} V+\partial_{t} V-r V=0 .
$$

Let $x_{i}=\ln S^{i}$. Equation (3) becomes

$$
\frac{1}{2} \sum_{i, j=1}^{n}\left(\sigma \sigma^{*}\right)_{i j} \partial_{x_{i} x_{j}} V+b \nabla V+\partial_{t} V-r V=0
$$

or

$$
\partial_{t} V+\frac{1}{2} \nabla_{x}^{*} A \nabla_{x} V+b^{*} \nabla_{x} V-r V=0
$$

where $b$ is a vector defined by

$$
b_{i}=r-\frac{1}{2} \sum_{i=1}^{n} \sigma_{i i}^{2}, i=1, \cdots, n .
$$

By variable substitution, $y=B x$. Note that $\nabla_{x}=B^{*} \nabla_{y}$. Equation (5) becomes

$$
\partial_{t} V+\frac{1}{2} \nabla_{y}^{*}\left(B A B^{*}\right) \nabla_{y} V+(B b)^{*} \nabla_{y} V-r V=0 .
$$

Because $A$ is a symmetric matrix, there exists an orthogonal transformation $B$ such that

$$
B A B^{*}=\Lambda=\left[\begin{array}{cccc}
\lambda_{1} & 0 & \cdots & 0 \\
0 & \lambda_{2} & \cdots & 0 \\
\vdots & \vdots & \ddots & \vdots \\
0 & 0 & \cdots & \lambda_{n}
\end{array}\right]
$$

where $\lambda_{1}, \cdots, \lambda_{n}$ are characteristic values of matrix $A$, and $\xi_{i}$ is the characteristic vector corresponding to $\lambda_{i}$. It is obvious that we can have

$$
B=\left[\begin{array}{c}
\xi_{1}^{*} \\
\xi_{2}^{*} \\
\vdots \\
\xi_{n}^{*}
\end{array}\right]=\left[\begin{array}{cccc}
\xi_{11} & \xi_{12} & \cdots & \xi_{1 n} \\
\xi_{21} & \xi_{22} & \cdots & \xi_{2 n} \\
\vdots & \vdots & \ddots & \vdots \\
\xi_{n 1} & \xi_{n 2} & \cdots & \xi_{n n}
\end{array}\right]
$$

Under the substitution $y=B x$, Equation (7) becomes

$$
\partial_{t} V+\frac{1}{2} \sum_{i=1}^{n} \lambda_{i} \frac{\partial^{2} V}{\partial y_{i}^{2}}+\sum_{i=1}^{n} \xi^{*} b \frac{\partial V}{\partial y_{i}}-r V=0 .
$$

Next we take $V=e^{\omega^{*} y+\beta(T-t)} u$, where $\beta$ is a constant and $\omega$ is a $n$-dimension vector, which will be confirmed later. Hence, we obtain

$$
\partial_{t} u+\frac{1}{2} \sum_{i=1}^{n}\left[\lambda_{i} \omega_{i}+\xi^{*} b\right] \frac{\partial u}{\partial y}+\frac{1}{2} \sum_{i=1}^{n} \lambda_{i} \frac{\partial^{2} u}{\partial y_{i}^{2}}-\left[r+\beta-\frac{1}{2} \omega^{*} \Lambda \omega-b^{*} B^{*} \omega\right] u=0 .
$$

Assume the following:

$$
\omega=-\Lambda^{-1} B b
$$


and

$$
\begin{aligned}
\beta & =-r+\frac{1}{2} \omega^{*} \Lambda \omega+b^{*} B^{*} \omega \\
& =-r+\frac{1}{2}(B b)^{*} \Lambda^{-1} \Lambda \Lambda^{-1}(B b)-b^{*} B^{*} \Lambda^{-1} B b \\
& =-r-\frac{1}{2}(B b)^{*} \Lambda^{-1}(B b)
\end{aligned}
$$

Equation (10) becomes

$$
\partial_{t} u=-\frac{1}{2} \sum_{i=1}^{n} \lambda_{i} \frac{\partial^{2} u}{\partial y_{i}^{2}}
$$

\section{Harnack Inequality and No-Arbitrage Analysis}

\subsection{Harnack Inequality}

Harnack inequality is used in many fields, the most important of which are those of parabolic differential equations [15] and nonlinear parabolic equations [16]. Carciola et al. [3] proved the Harnack inequality of Kolmogorov equations by using the method of optimal control, and they obtained the optimal Harnack constant. However, the works of Carciola et al. are heavily dependent on the fundamental solution of the Kolmogorov operator. Once the fundamental solution is unknown, how can one prove the Harnack inequality? For convenience and comparison, we also consider the Kolmogorov operator equation by adopting the argument in [17] to prove the Harnack inequality. As we know, the Kolmogorov operator [18] is expressed generally as

$$
L:=\frac{1}{2} \sum_{i, j=1}^{n} a_{i j}(t) \partial_{x_{i} x_{j}}+\langle b(t)+B(t) x, \nabla\rangle+\partial_{t},(t, x) \in \mathbb{R}^{n+1} .
$$

Let $f$ be a positive solution to $L f=0$, according to Equation (12), the Kolmogorov equation is equivalent to

$$
\partial_{t} f=-\frac{1}{2} \sum_{i=1}^{n} \lambda_{i} \frac{\partial^{2} f}{\partial y_{i}^{2}}
$$

Set

$$
\triangle_{\lambda} u=\lambda_{i} \nabla_{i} \nabla_{i} u, \quad|\nabla u|_{\lambda}^{2}=\lambda_{i} \nabla_{i} u \cdot \nabla_{i} u .
$$

Let $u=\log f$. We then have

$$
u_{t}=-\triangle_{\lambda} u-|\nabla u|_{\lambda}^{2} .
$$

In this paper, we will mainly consider the following Harnack quantity:

$$
H:=-\alpha \triangle_{\lambda} u-\beta|\nabla u|_{\lambda}^{2}+\phi .
$$

$\alpha, \beta \in R, \phi: \mathbb{R}^{n} \times[0, \infty)$ will be suitably chosen later.

Lemma 1. If $H$ is defined as in Equation (16), then

$$
\begin{aligned}
H_{t}= & -\triangle_{\lambda} H-2 \nabla H \cdot \nabla_{\lambda} u+\alpha\left[2 \sum_{i, j=1}^{n} \lambda_{i} \nabla_{j} \nabla_{i} u \cdot \lambda_{j} \nabla_{j} \nabla_{i} u-\triangle_{\lambda^{\prime}} u\right] \\
& -\beta\left[2 \sum_{i, j=1}^{n} \lambda_{i} \nabla_{j} \nabla_{i} u \cdot \lambda_{j} \nabla_{j} \nabla_{i} u+|\nabla u|_{\lambda^{\prime}}^{2}\right]+\triangle_{\lambda} \phi+2 \nabla \phi \cdot \nabla_{\lambda} u+\phi_{t}
\end{aligned}
$$


Proof. By a direct calculation, we obtain

$$
\partial_{t}\left(|\nabla u|_{\lambda}^{2}\right)=-2 \nabla_{\lambda} u \cdot \nabla \triangle_{\lambda} u-2 \nabla_{\lambda} u \cdot \nabla|\nabla u|_{\lambda}^{2}+|\nabla u|_{\lambda^{\prime}}^{2}
$$

and

$$
\partial_{t}\left(\triangle_{\lambda} u\right)=\triangle_{\lambda^{\prime}} u-\triangle_{\lambda}\left(\triangle_{\lambda} u\right)-\triangle_{\lambda}\left(|\nabla u|_{\lambda}^{2}\right)
$$

In addition,

$$
\triangle_{\lambda}\left(|\nabla u|_{\lambda}^{2}\right)=2 \sum_{i, j=1}^{n} \lambda_{i} \nabla_{j} \nabla_{i} u \cdot \lambda_{j} \nabla_{j} \nabla_{i} u+2 \nabla_{\lambda} u \cdot \nabla \triangle_{\lambda} u
$$

We then have

$$
\begin{aligned}
H_{t}= & \alpha \triangle_{\lambda}\left(\triangle_{\lambda} u\right)+\alpha \triangle_{\lambda}|\nabla u|_{\lambda}^{2}-\alpha \triangle_{\lambda^{\prime}} u+2 \beta \nabla_{\lambda} u \cdot \nabla\left(\triangle_{\lambda} u\right) \\
& +2 \beta \nabla\left(|\nabla u|_{\lambda}^{2}\right) \cdot \nabla_{\lambda} u-\beta|\nabla u|_{\lambda^{\prime}}^{2}+\phi_{t} .
\end{aligned}
$$

After simplifying Equation (19), we obtain

$$
\begin{aligned}
H_{t}= & -\triangle_{\lambda} H-2 \nabla H \cdot \nabla_{\lambda} u+\alpha\left[2 \sum_{i, j=1}^{n} \lambda_{i} \nabla_{j} \nabla_{i} u \cdot \lambda_{j} \nabla_{j} \nabla_{i} u-\triangle_{\lambda^{\prime}} u\right] \\
& -\beta\left[2 \sum_{i, j=1}^{n} \lambda_{i} \nabla_{j} \nabla_{i} u \cdot \lambda_{j} \nabla_{j} \nabla_{i} u+|\nabla u|_{\lambda^{\prime}}^{2}\right]+\triangle_{\lambda} \phi+2 \nabla \phi \cdot \nabla_{\lambda} u+\phi_{t} .
\end{aligned}
$$

This completes the proof of Lemma 1.

For convenience, we assume

$Q_{1} . \quad \frac{1}{N}<\frac{\partial^{m+l} u}{\partial y_{i}^{m} \partial y_{j}^{l}}<1(i, j=1, \cdots, n)$, where $N>1$, the integers $m, l \geq 0$ satisfy $1 \leq m+l \leq 4$.

$Q_{2} . \quad 5 \sum_{i=1}^{n} \lambda_{i}^{2}+10 \sum_{i \neq j}^{n} \lambda_{i} \lambda_{j} \leq \frac{\alpha}{N \beta} \sum_{i=1}^{n} \lambda_{i}^{\prime}$.

Lemma 2. Assume that $Q_{1}$ and $Q_{2}$ are tenable. The following then holds:

$$
\alpha \triangle_{\lambda}\left(\triangle_{\lambda} u\right)+\beta \triangle_{\lambda}|\nabla u|_{\lambda}^{2} \leq \alpha \triangle_{\lambda^{\prime}} u
$$

Theorem 1. Suppose $f(x, t)$ is a positive solution to Equation (14). If $Q_{1}$ and $Q_{2}$ hold, $\alpha, \beta$, a satisfy

$$
0<\alpha<\beta,
$$

and

$$
a \geq \frac{n \alpha^{2}}{2(\beta-\alpha)}>0
$$

Assume $u=\log f$. Then the following holds:

$$
H^{0} \equiv-\alpha \triangle_{\lambda} u-\beta|\nabla u|_{\lambda}^{2}-\frac{a}{t} \leq 0
$$

for all $t$. 
Proof. We choose $\phi_{R}(y, t)$ defined on the n-rectangle denoted by $R \subset \mathbb{R}^{n}$, which is made up of a Cartesian product of $n$ intervals $\left[p_{i}, q_{i}\right]$, so that $\phi_{R} \rightarrow-\infty$ if $y_{i} \rightarrow p_{i}, q_{i}$ or if $t \rightarrow 0$. More precisely, we can take

$$
\phi_{R}(y, t)=-\frac{a}{t}-\sum_{k=1}^{n} \lambda_{k}\left(\frac{b}{\left(y_{k}-p_{k}\right)^{2}}+\frac{b}{\left(q_{k}-y_{k}\right)^{2}}\right)
$$

for $t>0$ and $y=\left(y_{1}, \cdots, y_{n}\right) \in R=\Pi_{1}^{n}\left[p_{i}, q_{i}\right]$. The corresponding Harnack quantity is

$$
H^{R}=-\alpha \triangle_{\lambda} u-\beta|\nabla u|_{\lambda}^{2}+\phi_{R}(y, t) .
$$

It is obvious that $H^{R} \rightarrow H^{0}$, while the rectangle $R=\Pi_{1}^{n}\left[p_{i}, q_{i}\right] \rightarrow \mathbb{R}^{n}$, and $H^{R}<0$ for small $t(t \rightarrow 0)$.

Next, we will illustrate $H^{R}<0$ by reduction to absurdity. Assume that $H^{R}$ arrives at zero for the first time at point $t_{0}$ and point $y_{0} \in R$, that is, $H^{R}\left(y_{0}, t_{0}\right)=0$. Then, at point $\left(y_{0}, t_{0}\right)$, by maximum principle we have

$$
\left(H^{R}\right)_{t} \geq 0, \nabla H^{R}=0, \triangle_{\lambda} H^{R} \leq 0
$$

and

$$
\triangle_{\lambda} u=-\frac{1}{\alpha}\left(\beta|\nabla u|_{\lambda}^{2}-\phi_{R}\right)
$$

By applying Lemma 1 and the inequality $\sum_{i, j=1}^{n} \lambda_{i} \nabla_{j} \nabla_{i} u \cdot \lambda_{j} \nabla_{j} \nabla_{i} u \geq \frac{1}{n}\left(\triangle_{\lambda} u\right)^{2}$, we have

$$
\begin{aligned}
0 \leq & -\triangle_{\lambda} H^{R}-2 \nabla H^{R} \cdot \nabla_{\lambda} u+2(\alpha-\beta) \sum_{i, j=1}^{n} \lambda_{i} \nabla_{j} \nabla_{i} u \cdot \lambda_{j} \nabla_{j} \nabla_{i} u \\
& -\alpha \triangle_{\lambda^{\prime}} u-\beta|\nabla u|_{\lambda^{\prime}}^{2}+\triangle_{\lambda} \phi_{R}+2 \nabla \phi_{R} \cdot \nabla_{\lambda} u+\left(\phi_{R}\right)_{t}
\end{aligned}
$$

With Lemma 2, we have

$$
-\triangle_{\lambda} H^{R}-\alpha \triangle_{\lambda^{\prime}} u+\triangle_{\lambda} \phi_{R}=\alpha \triangle_{\lambda}\left(\triangle_{\lambda} u\right)+\beta \triangle_{\lambda}|\nabla u|_{\lambda}^{2}-\alpha \triangle_{\lambda^{\prime}} u \leq 0 .
$$

By Inequality (20) and by setting $Y=|\nabla u|_{\lambda}^{2}$, Inequality (24) becomes

$$
\begin{aligned}
0 \leq & \frac{2(\alpha-\beta)}{n \alpha^{2}} \beta^{2} Y^{2}+\frac{2(\alpha-\beta)}{n \alpha^{2}} \phi_{R}^{2}-\frac{4(\alpha-\beta) \beta}{n \alpha^{2}} Y \phi_{R} . \\
& -\beta|\nabla u|_{\lambda^{\prime}}^{2}+2 \nabla \phi_{R} \cdot \nabla_{\lambda} u+\left(\phi_{R}\right)_{t}
\end{aligned} .
$$

We now illustrate that the right-hand side is in fact negative, which will give us a contradiction. Calculating $-\frac{4(\alpha-\beta) \beta}{n \alpha^{2}} Y \phi_{R}$ and $2 \nabla_{\lambda} \phi_{R} \cdot \nabla u$, we obtain

$$
\begin{aligned}
& -\frac{4(\alpha-\beta) \beta}{n \alpha^{2}} Y \phi_{R}+2 \nabla \phi_{R} \cdot \nabla_{\lambda} u \\
& \leq-\frac{4(\alpha-\beta) \beta \phi_{R}}{n \alpha^{2}}\left(\left|\sqrt{\lambda}_{i} \nabla_{i} u\right|-\frac{n \alpha^{2}\left|\sqrt{\lambda}_{i} \nabla_{i} \phi_{R}\right|}{4(\alpha-\beta) \beta \phi_{R}}\right)^{2} . \\
& +\frac{4(\alpha-\beta) \beta \phi_{R}}{n \alpha^{2}} \cdot\left(\frac{n \alpha^{2}\left|\sqrt{\lambda}_{i} \nabla_{i} \phi_{R}\right|}{4(\alpha-\beta) \beta \phi_{R}}\right)^{2}
\end{aligned}
$$


Noting that $\phi_{R}<0$, we have

$$
-\frac{4(\alpha-\beta) \beta}{n \alpha^{2}} \Upsilon \phi_{R}+2 \nabla_{\lambda} \phi_{R} \cdot \nabla u \leq \frac{n \alpha^{2}\left|\sqrt{\lambda}_{i} \nabla_{i} \phi_{R}\right|^{2}}{4(\alpha-\beta) \beta \phi_{R}} .
$$

Thus, we arrive at

$$
0 \leq\left(\phi_{R}\right)_{t}+\frac{n \alpha^{2}\left|\sqrt{\lambda_{i}} \nabla_{i} \phi_{R}\right|^{2}}{4(\alpha-\beta) \beta \phi_{R}}+\frac{2(\alpha-\beta)}{n \alpha^{2}} \phi_{R}^{2} .
$$

We can deal with this by a direct calculation. It is easy to see that

$$
\left|\sqrt{\lambda}_{k} \nabla_{k} \phi_{R}\right|^{2}=\sum_{k=1}^{n} \lambda_{k}^{3}\left(\frac{2 b}{\left(y_{k}-p_{k}\right)^{3}}-\frac{2 b}{\left(q_{k}-y_{k}\right)^{3}}\right)^{2}
$$

and to observe that

$$
\frac{\left|\sqrt{\lambda_{k}} \nabla_{k} \phi_{R}\right|^{2}}{\phi_{R}} \geq-\sum_{k=1}^{n} \lambda_{k}^{2}\left(\frac{2 \sqrt{b}}{\left(y_{k}-p_{k}\right)^{2}}+\frac{2 \sqrt{b}}{\left(q_{k}-y_{k}\right)^{2}}\right)^{2} .
$$

We set the following:

$$
A:=\frac{2(\alpha-\beta)}{n \alpha^{2}}<0, \quad B:=\frac{n \alpha^{2}}{4(\alpha-\beta) \beta}<0 .
$$

To arrive at a contradiction, we choose $a$ as in Inequality (21) so that $A a^{2}+a \leq 0$. Next, plugging Inequalities (23) and (28) into Inequality (27), we obtain

$$
\begin{aligned}
0 & \leq A \sum_{k=1}^{n} \lambda_{k}^{2}\left(\frac{b}{\left(y_{k}-p_{k}\right)^{2}}+\frac{b}{\left(q_{k}-y_{k}\right)^{2}}\right)^{2}-B \sum_{k=1}^{n} \lambda_{k}^{2}\left(\frac{2 \sqrt{b}}{\left(y_{k}-p_{k}\right)^{2}}+\frac{2 \sqrt{b}}{\left(q_{k}-y_{k}\right)^{2}}\right)^{2} \\
& \leq\left[A b^{2}-4 B b\right] \sum_{k=1}^{n} \lambda_{k}^{2}\left(\frac{1}{\left(y_{k}-p_{k}\right)^{2}}+\frac{1}{\left(q_{k}-y_{k}\right)^{2}}\right)^{2}
\end{aligned}
$$

We conclude that it is sufficient to have

$$
A b^{2}-4 B b<0,
$$

which reduces to

$$
b>\frac{4 B}{A}
$$

In conclusion, $a$ and $b$ satisfy

$$
a \geq \frac{n \alpha^{2}}{2(\beta-\alpha)}, \quad b>\frac{n^{2} \alpha^{4}}{2(\alpha-\beta)^{2} \beta} .
$$

At this point we can observe that the right-hand side of Inequality (24) is in fact negative, which is a contradiction. It is impossible for $H^{R}$ to reach zero for the first time. Therefore, $H^{R}<0$, and it is obvious that $H^{0}<0$. Assume the solution exits in all of space $\mathbb{R}^{n}$, we can let $R \rightarrow \mathbb{R}^{n}$ so that $\phi_{R} \rightarrow-\frac{a}{t}$. This completes the proof of Theorem 1. 
Theorem 2. Let $f$ be a positive solution to Equation (14). Let $\gamma(t)=(y(t), t), t \in\left[t_{1}, t_{2}\right]$, be a space-time curve joining two given points $\left(y_{1}, t_{1}\right),\left(y_{2}, t_{2}\right) \in \mathbb{R}^{n} \times[0, \infty)$ with $0<t_{1}<t_{2}$. Assume further that $a \leq \frac{n \alpha}{2}$, $|\nabla u|<M(M>0)$ and $\beta=\alpha+\varepsilon(\varepsilon \rightarrow 0)$. We then have

$$
f\left(y_{2}, t_{2}\right) \leq f\left(y_{1}, t_{1}\right)\left(\frac{t_{2}}{t_{1}}\right)^{\frac{n}{2}} \exp \left[\frac{1}{2} M^{2}\left(t_{2}-t_{1}\right)+\frac{\left|y_{2}-y_{1}\right|^{2}}{2\left(t_{2}-t_{1}\right)}\right] .
$$

Proof. Assume $u=\log f$ in Equation (14). Then

$$
u_{t}=-\triangle_{\lambda} u-|\nabla u|_{\lambda}^{2}
$$

By the differential Harnack inequality expressed in Inequality (22), we have $H^{0} \leq 0$, so

$$
-\triangle_{\lambda} u \leq \frac{1}{\alpha}\left(\beta|\nabla u|_{\lambda}^{2}+\frac{a}{t}\right) .
$$

We then compute $u$ along $\gamma$ :

$$
\begin{aligned}
\frac{d}{d t}[u(y(t), t)] & =\nabla u \cdot \dot{y}+u_{t} \\
& =\nabla u \cdot \dot{y}-\triangle_{\lambda} u-|\nabla u|_{\lambda}^{2} \\
& \leq \frac{1}{\alpha}\left(\beta|\nabla u|_{\lambda}^{2}+\frac{a}{t}\right)-|\nabla u|_{\lambda}^{2}+\nabla u \cdot \dot{y} \\
& =\left(\frac{\beta}{\alpha}-1\right)|\nabla u|_{\lambda}^{2}+\nabla u \cdot \dot{y}+\frac{a}{\alpha t} \\
& \leq\left(\frac{\beta}{\alpha}-1\right)|\nabla u|_{\lambda}^{2}+\frac{1}{2}|\nabla u|^{2}+\frac{1}{2}|\dot{y}|^{2}+\frac{a}{\alpha t} \\
& \leq \frac{1}{2} M^{2}+\frac{1}{2}|\dot{y}|^{2}+\frac{n}{2 t}
\end{aligned}
$$

Integrating the above inequality expressed in Inequality (30) along $\gamma$, and taking the infimum over all the space-time paths,

$$
u\left(y_{2}, t_{2}\right)-u\left(y_{1}, t_{1}\right) \leq \inf _{\gamma(t)=(y(t), t)} \int_{t_{1}}^{t_{2}}\left[\frac{1}{2} M^{2}+\frac{1}{2}|\dot{y}|^{2}+\frac{n}{2 t}\right] d t .
$$

Recalling that $u=\log f$, we arrive at Theorem 2 .

Remark 1. Theorem 2 compares any two values of $f$ in Equation (14). Recall the B-S equation, where we could compare the portfolio values at any two times. In Section 3.2, we will consider a two-period financial model and compare the portfolio values at the beginning and the end of the period by Theorem 2.

\subsection{No-Arbitrage Analysis}

Consider a single stage financial market with $n$ assets and $s$ elementary states of the world. The initial value of $n$ assets are $S_{0}^{1}, S_{0}^{2}, \cdots, S_{0}^{n}$, and their future values are $S^{1}, S^{2}, \cdots, S^{n} \in \mathcal{M}^{s}$, where $\mathcal{M}^{s}$ is a collection of contingent claims with $s$ status. A general strategy is described by $\theta=\left(\theta_{1}, \theta_{2}, \cdots, \theta_{n}\right)^{T} \in \Theta^{n}$. The cost of this strategy is

$$
\begin{aligned}
V_{0} & =\theta_{1} S_{0}^{1}+\theta_{2} S_{0}^{2}+\cdots+\theta_{n} S_{0}^{n} \\
& =\left(S_{0}^{1}, S_{0}^{2}, \cdots, S_{0}^{n}\right) \cdot\left(\theta_{1}, \theta_{2}, \cdots, \theta_{n}\right)^{T} . \\
& =S_{0}^{T} \cdot \theta \in \mathbb{R}
\end{aligned}
$$


At the end of the period, the portfolio value is

$$
\begin{aligned}
V_{1}(\theta)= & S \theta=\theta_{1} S^{1}+\theta_{2} S^{2}+\cdots+\theta_{n} S^{n} \\
= & \left(\begin{array}{cccc}
S^{11} & S^{21} & \ldots & S^{n 1} \\
S^{12} & S^{22} & \cdots & S^{n 2} \\
\vdots & \vdots & \ddots & \vdots \\
S^{1 s} & S^{2 s} & \cdots & S^{n s}
\end{array}\right) \cdot\left(\begin{array}{c}
\theta_{1} \\
\theta_{2} \\
\vdots \\
\theta_{n}
\end{array}\right) \hat{=} \mathcal{S} \cdot \theta \in \mathcal{M}^{s}
\end{aligned}
$$

where $\mathcal{S}$ is a pay-off matrix. We denote the single stage financial market by $\left(\mathcal{M}^{s}, \Theta^{n}, \mathcal{S}\right)$.

Definition 1 (No-arbitrage). For the market $\left(\mathcal{M}^{s}, \Theta^{n}, \mathcal{S}\right)$, if there exists a strategy $\theta^{\prime} \in \Theta^{n}$, such that $V_{0}\left(\theta^{\prime}\right) \leq 0$ and $V_{1}\left(\theta^{\prime}\right)>0$, we say that the market $\left(\mathcal{M}^{s}, \Theta^{n}, \mathcal{S}\right)$ has the first kind of arbitrage. If there exists a strategy $\theta^{\prime \prime} \in \Theta^{n}$, such that $V_{0}\left(\theta^{\prime \prime}\right)<0$ and $V_{1}\left(\theta^{\prime \prime}\right)=0$, we say that the market $\left(\mathcal{M}^{s}, \Theta^{n}, \mathcal{S}\right)$ has the second kind of arbitrage.

We say that there is no arbitrage opportunity in the market $\left(\mathcal{M}^{s}, \Theta^{n}, \mathcal{S}\right)$ if there is neither the first arbitrage opportunity nor the second arbitrage opportunity.

For details regarding the financial background, we refer the reader to $[19,20]$.

Proposition 1. Consider the self-financing portfolio mentioned in Equation (1), which defined [0,T]. By Theorem 2, we have

$$
V\left(t, S_{t}\right) \leq e^{r t} H\left(S_{0}, S_{t}, t\right) V\left(0, S_{0}\right), \quad 0 \leq t<T
$$

where

$$
\begin{aligned}
H\left(S_{0}, S_{t}, t\right) & =\left(\frac{T}{T-t}\right)^{\frac{n}{2}} \exp \left[\frac{\left|S_{t}-S_{0}\right|^{2}}{2 t}+\frac{M^{2} t}{2}\right] \\
& =\left(\frac{T}{T-t}\right)^{\frac{n}{2}} \exp \left[\frac{1}{2 t} \sum_{i=1}^{n}\left(S_{t}^{i}-S_{0}^{i}\right)^{2}+\frac{M^{2} t}{2}\right]
\end{aligned}
$$

Proof. In fact, one can only take $V\left(t, S_{t}\right) \hat{=} f\left(t, y_{t}\right), S_{t} \hat{=} y_{t}$ in Theorem 2. This ends the proof of Proposition 1.

Corollary 1. Formula (31) illustrates that it is of no-arbitrage in the self-financing market $\left(\mathcal{M}^{s}, \Theta^{n}, \mathcal{S}\right)$.

\subsection{Example}

Consider a market with a risk asset $S_{t}$ and a risk-free asset $B_{t}$. Suppose the assets are given by

$$
\begin{gathered}
d S_{t}=r S_{t} d t+\sigma_{0} S_{t} d W_{t} \\
d B_{t}=r_{t} B_{t} d t
\end{gathered}
$$

Set $b=r-\frac{1}{2} \sigma_{0}^{2}$. As a consequence of Corollary 1 , it is obvious that the market is of no-arbitrage if the portfolio value satisfies the following inequality:

$$
V\left(S_{t}, t\right) \leq e^{r t} H\left(S_{0}, S_{t}, t\right) V\left(S_{0}, 0\right), \quad 0 \leq t<T
$$

where

$$
H\left(S_{0}, S_{t}, t\right)=\left(\frac{T}{T-t}\right)^{\frac{1}{2}} \exp \left[\frac{S_{0}^{2}}{2 t}\left(\exp \left\{\int_{0}^{t} \sigma_{0} d W_{s}+b t\right\}-1\right)^{2}+\frac{M^{2} t}{2}\right]
$$




\section{Conclusions}

This paper gives the no-arbitrage analysis by a Harnack inequality for the option pricing equation with gradient arguments. In particular, we do not need to derive the fundamental solution of this Kolmogorov equation. Therefore, we could arrive at the no-arbitrage analysis if the fundamental solution of an option pricing equation is unknown.

Author Contributions: All authors contributed equally and significantly this paper. All authors read and approved the final manuscript.

Funding: This research was funded by National Natural Science Foundation of China (No. 11871275, No. 11371194).

Conflicts of Interest: The authors declare no conflict of interest.

\section{References}

1. Kawabi, H. The Parabolic Harnack Inequality for the Time Dependent Ginzburg-Landau Type SPDE and its Application. Potential Anal. 2005, 22, 61-84. [CrossRef]

2. Arnaudon, M.; Thalmaier, A.; Wang, F. Harnack inequality and heat kernel estimates on manifolds with curvature unbounded below. Bull. Sci. Math. 2006, 130, 223-233. [CrossRef]

3. Carciola, A. Harnack inequality and no-arbitrage bounds for self-financing portfolios. Bol. Soc. Esp. Mat. Apl. 2009, 49, 15-27.

4. Wang, F. Harnack Inequality and Applications for Stochastic Generalized Porous Media Equations. Ann. Probab. 2007, 35, 1333-1350. [CrossRef]

5. Bass, R.F.; Levin, D.A. Harnack inequalities for jump processes. Potential Anal. 2002, 17, 375-388. [CrossRef]

6. Harrison, J.M.; Pliska, S.R. Martingales and stochastic integrals in the theory of continuous trading. Stoch. Process. Their Appl. 1981, 11, 215-260. [CrossRef]

7. Cox, J.C.; Ross, S.A. The valuation of options for alternative stochastic processes. J. Financ. Econ. 1976, 3, 145-166. [CrossRef]

8. Deng, X.; Li, Z.; Wang, S.; Yang, H. Necessary and Sufficient Conditions for Weak No-Arbitrage in Securities Markets with Frictions. Ann. Oper. Res. 2005, 133, 265-276. [CrossRef]

9. Sandhu, R.; Georgiou, T.; Tannenbaum, A. Market Fragility, Systemic Risk, and Ricci Curvature. arXiv 2015, arXiv:1505.05182.

10. Brody, D.C.; Hughston, L.P. Interest Rates and Information Geometry. R. Soc. 2011, 457, 1343-1363. [CrossRef]

11. Young, K. Foreign exchange market as a lattice gauge theory. Am. J. Phys. 1999, 67, 862-868. [CrossRef]

12. Ilinski, K. Physics of Finance: Gauge Modelling in Non-Equilibrium Pricing; Wiley: Hoboken, NJ, USA, 2001.

13. Farinelli, S. Geometric Arbitrage Theory and Market Dynamics. J. Geom. Mech. 2015, 7, 431-471. [CrossRef]

14. Choi, Y.H. Curvature Arbitrage. Ph.D. Thesis, University of Iowa, Iowa City, IA, USA, 2007.

15. Moser, J. A Harnack inequality for parabolic differential equations. Commun. Pure Appl. Math. 1964, 17, 101-134. [CrossRef]

16. Huang, G.; Huang, Z.; Li, H. Gradient estimates and differential Harnack inequalities for a nonlinear parabolic equation on Riemannian manifolds. Ann. Glob. Anal. Geom. 2013, 43, 209-232. [CrossRef]

17. Cao, X. Harnack estimate for the endangered species equation. Proc. Am. Math. Soc. 2015, 143, 4537-4545. [CrossRef]

18. Garofalo, N.; Lanconelli, E. Level sets of the fundamental solution and Harnack inequality for degenerate equations of Kolmogorov type. Trans. Am. Math. Soc. 1990, 321, 775-792. [CrossRef]

19. Barucci, E.; Fontana, C. Financial Markets Theory; Springer: Berlin, Germany, 2017.

20. Delbaen, F.; Schachermayer, W. The Mathematics of Arbitrage; Springer: Berlin, Germany, 2006.

(C) 2018 by the authors. Licensee MDPI, Basel, Switzerland. This article is an open access article distributed under the terms and conditions of the Creative Commons Attribution (CC BY) license (http://creativecommons.org/licenses/by/4.0/). 